\title{
A novel scale to assess psychosis in patients with parkinson's disease
}

William G. Ondo ${ }^{1 *}$, Sana Sarfaraz ${ }^{1}$ and MinJae Lee Le, $^{2,3}$

\begin{abstract}
Background: Organic psychosis effects up to $70 \%$ of patients with PD at some point yet no widely accepted scale for this entity exists.

Methods: We developed a 10 question PD specific psychosis severity scale that we feel has good content validity. It asks about the presence, severity, frequency, and consequences of the hallucinations (visual, auditory, olfactory) and delusions.

Results: Fifty different PD patients with psychosis and 25 PD patients without psychosis were included, and serial information was available in 21 of those encounters with psychosis. In psychosis subjects, results were normally distributed: mean 17.23 (SD =6.30). In those without psychosis $14 \%$ scored $>0$, mean 0.36 [range0-7]. The intra-rater, inter-class correlation coefficient was excellent ( $N=21$ pairs of observations seven days apart, ICC $=0.87$ ). Inter-rater reliability (two different raters, $\mathrm{N}=46$ pairs) was outstanding for the entire group, ICC $=0.92$ ). As expected visual hallucinations were most common (mean $=3.13$ ). The presence of delusions was associated with greater total scores.
\end{abstract}

Conclusions: This scale, specifically designed for PD psychosis is easy to administer and has impressive metrics.

Keywords: Parkinson's disease, Psychosis, Hallucinations, Delusions, Scale, Metrics

\section{Background}

Psychosis in Parkinson's disease (PD) affects up to $70 \%$ of patients, and causes tremendous morbidity [1]. The phenotype is much different than psychosis associated with schizophrenia or delirium. Although many different hallucinations, illusions, and delusions are reported in $\mathrm{PD}$, the majority of episodes are visual hallucinations (usually "benign" content of silent people or animals), and persecutory/infidelity delusions, which are usually more problematic [1].

Despite the prevalence and importance of PD psychosis, no widely accepted scale exists. A Movement Disorder Task Force evaluated scales used to assess psychosis in $\mathrm{PD}$, and concluded that a novel PD specific scale is needed [2]. Several small scales designed specifically for PD were felt to be insufficiently inclusive or poorly validated [3, 4], whereas better known and validated psychosis scales were not designed for PD psychosis and were felt to have poor content validity [5-7].

\footnotetext{
* Correspondence: William.Ondo@uth.tmc.edu

'Department of Neurology, Methodist Neurological Institute, 6560 Fannin, Ste 802, Houston, TX 77030, USA

Full list of author information is available at the end of the article
}

The lack of a disease specific severity scale has probably hampered efforts to test treatments for PD psychosis. To this day only clozapine and pimavanserin have compelling published efficacy data [8, 9]. To better quantify PD psychosis and aid in future therapeutic trials, we designed a PD specific psychosis scale and undertook psychometric evaluations.

\section{Methods}

The main goals for this scale development were content validity (based on the most common psychosis symptoms in the literature and experience, as there is no gold standard scale to compare), ease of use (10-15 minutes), inter-rater validation to include both physicians and nonphysicians, and intra-rater validity measured over time. It is not a specifically designed quality of life scale, although questions 6-10 investigate how the psychosis would likely effect quality of life (insight, affective consequences and actions, and impact on family), nor is it designed as a screening tool. The questions evolved over 10 years and included patient and family input. Earlier non-validated 
versions of the scale have been used in previous clinical trials $[10,11]$.

The study received a waiver from full consent from the University of Texas Health Science Center Institutional Review Board. All PD patients were recruited from a movement disorder center. We included PD patients with psychosis, based on a rating of $\geq 1$ on UPDRS question \#2, as determined by interview with the investigator, and a comparator group of PD patients without psychosis, score $=0$. There were no formal exclusion criteria other than complete inability to participate. We intentionally allowed subjects with clinical dementia but in all cases motor symptoms preceded cognitive symptoms by more than a year [12]. Dementia was diagnosed if patients had a chart documented diagnosis of dementia and/or were taking acetylcholinesterase inhibitors for dementia. All consecutively seen psychosis patients seen in our tertiary referral center were included, but the control group was a convenience sample from the same clinic seen over the same period.

The first five questions identify the type of hallucination (visual, auditory, olfactory, sense of presence) or delusion [Fig. 1: Scale]. The second five questions further quantify the intensity, frequency, insight and impact of the worst psychotic feature on the life of the patient and family. The source of the information (patient/family/both) is documented. Since insight varies among patients, some latitude in question semantics is allowed and we did not include a specific question on how the psychosis affects only the patient's quality of life. [Additional file 1: Instructions] The final answer (0-4 for each question) is the opinion of the interviewer. It is not self-administered.

The patients were interviewed by an experienced physician and an inexperienced coordinator who had just started working with PD patient, at least 15 minutes apart (inter-rater assessments). Physician interview preceded the "inexperienced" interview in all cases. Intra-rater reliability (test-retest) was also tested at a second point in time $7+/-3$ days after the first administration, in patients who did not require therapeutic intervention (change in medications) prior to then. This was done by a single interviewer (WO).

The range of score for each specific question is $0-4$ and the total score simply adds all 10 questions. Weighted kappa statistics were calculated for inter and intra-rater reliability on the scale ranged $0-4$ for each question, and intra-class correlation coefficients were calculated for inter- and intra-rater reliability on total score and individual questions. We used the mixed effect model to account for correlation within subjects. Poisson and linear model were conducted for patients with/without psychosis and with psychosis respectively because the total score for those with psychosis is normally distributed but not for those with non-psychosis [Fig. 2]. Descriptive statistics are also presented. Analyses were performed using SAS 9.3 (SAS Institute Inc, Cary, NC) at a statistical significance level of 0.05 .

\section{Results}

On average the questionnaire required a 10 min interview and family input was obtained in $84.8 \%$ of all interviews. Inter-rater testing was completed on 46 different subjects with psychosis and 25 PD patients without psychosis. One subject was excluded because psychosis status was not definitively marked, leaving 49 , and three psychosis subjects had intra-rater serial data, but not inter-rater data. The mean age for all 75 subjects (51 male) was $70.0 \pm 10.8$ years and duration of PD motor features was $9.4 \pm 5.1$ years. Dementia was diagnosed in 26 of the 75 total subjects (34.7\%). The PD without psychosis and PD with psychosis groups had similar age $(68.8 \pm 9.1$ vs $70.6 \pm 11.6$ years $)$, duration of PD $8.5 \pm 5.0$ vs $9.9 \pm 5.1$ years), percent that were male (68\% vs. $70 \%)$, and percent that were demented (36.0 \% vs. 34.0).

For those with psychosis, the results were normally distributed with a mean score of $17.23 \pm 6.30$, [range: 3-32]. Only 7 of 50 questionnaires from subjects without psychosis scored greater than 0 , and only 1 scored $>3$ [Fig. 2]. The inter-rater reliability was excellent for the entire group (71 pair including psychotic and non-psychotic subjects, ICC $=0.92$ ). For just those with psychosis (46 pairs) the ICC was 0.87 . The intra-rater, inter-class correlation coefficient (time 1 vs. time 2 with same administrator) was excellent, $\mathrm{N}=21$ pairs of observations, $\mathrm{ICC}=0.88$. As expected, visual hallucinations were most common $($ mean $=3.13$ ), followed by sensing a presence (mean $=2.06$ ), auditory $($ mean $=1.16)$, and olfactory $($ mean $=0.29) \quad[$ Table 1]. A delusion (question \#5) was scored as $>0$ in 36/100 PD psychosis questionnaires. The total score in these cases with any delusion was much higher than those with pure hallucinations without delusions $($ mean $=22.51$ vs. 14.49 , $p<0.001$, powered by higher responses in questions \#6-10 (mean of 12.53 vs $7.73, p<0.001$ ).

\section{Discussion}

We report very good intra-rater reliability and excellent inter-rater reliability on a 10 question scale designed specifically for PD associated psychosis. Several content features warrant comment. We did not include a separate question on illusions because subjects often have difficulty differentiating these from true hallucinations so it could be "double scored". We instruct that illusions be included with hallucinations (almost always visual). We did not include tactile hallucinations. These have been reported [13, 14], but in our experience are essentially impossible to differentiate from "actual" sensations, which are very common in parkinsonism, and do not 
1. How frequently do you see things that other people do not see (hallucinations)?

0 . Never

1. Rarely

2. Occasionally (about once per week)

3. Frequently (more than three times per week, $50 \%$ of days)

4. Almost always (every day)

2. How frequently do you smell things that other people do not smell, and that possibly are not there (hallucinations)?

0 . Never

1. Rarely

2. Occasionally (about once per week)

3. Frequently (more than three times per week, $50 \%$ of days)

4. Almost always (every day)

3. How frequently do you hear things that other people do not hear, and that possibly are not there (hallucinations)?

0 . Never

1. Rarely (less than once per week)

2. Occasionally (at least once per week)

3. Frequently (more than three times per week, $50 \%$ of days)

4. Almost always (more than once per day)

4. How frequently do you sense a presence that other people do not sense, for example someone is behind you but you don't actually see them (hallucinations) ?

0 . Never

1. Rarely

2. Occasionally (about once per week)

3. Frequently (more than three times per week, $50 \%$ of days)

4. Almost always (more than once per day)

5. Do you ever feel cheated or persecuted by people, examples include a spouse that is unfaithful or that some people are trying to harm you for no reason?

0 . No

1. Occasionally I feel this but I know it is not true

2. Often I feel this but I know it is not true

3. Occasionally I feel this and am sure it is true

4. Often I feel this and am sure it is true

6 . When you do, for example, see, hear smell, sense a presence, or feel cheated, for how long will it typically last during that episode? (score the worst of Questions 1-5)

0 . I do not see, hear of sense any presence

1. just a second

2. seconds (2-59 seconds)

3. minutes (1-59 minutes)

4. hours ( $>59$ minutes)

7. Can you tell that these things (hallucinations/delusions) [ask about whichever is most prominent] are not real?

0 . I am not having any now

1. I have them, but can always tell they are not real

2. after talking to other people who say they are not real, I am convinced they are definitely not real

3. after talking to other people who say they are not real, I am not sure if they are real

4. after talking to other people who say they are not real, I still definitely feel that they are real

8. When you have the hallucinations, do the hallucinations ever threaten you in any way ? This means their actions against you, not just the fact that they are there.

0 . I don't have any hallucinations now

1. Never

2. Sometimes

3. Usually

4. Always

9. Do you try to communicate or interact with the hallucinations ?

0 . I don't have any hallucinations now

1. Never

2. Sometimes

3. Usually

4. Always

10. How upset or concerned is your family about the hallucinations. If your family does not know about them, how upset do you think they would be about the hallucinations ?

0 . Not concerned at all

1. Mildly concerned

2. Moderately concerned

3. Markedly concerned

4. Extremely concerned

Fig. 1 Actual Scale 


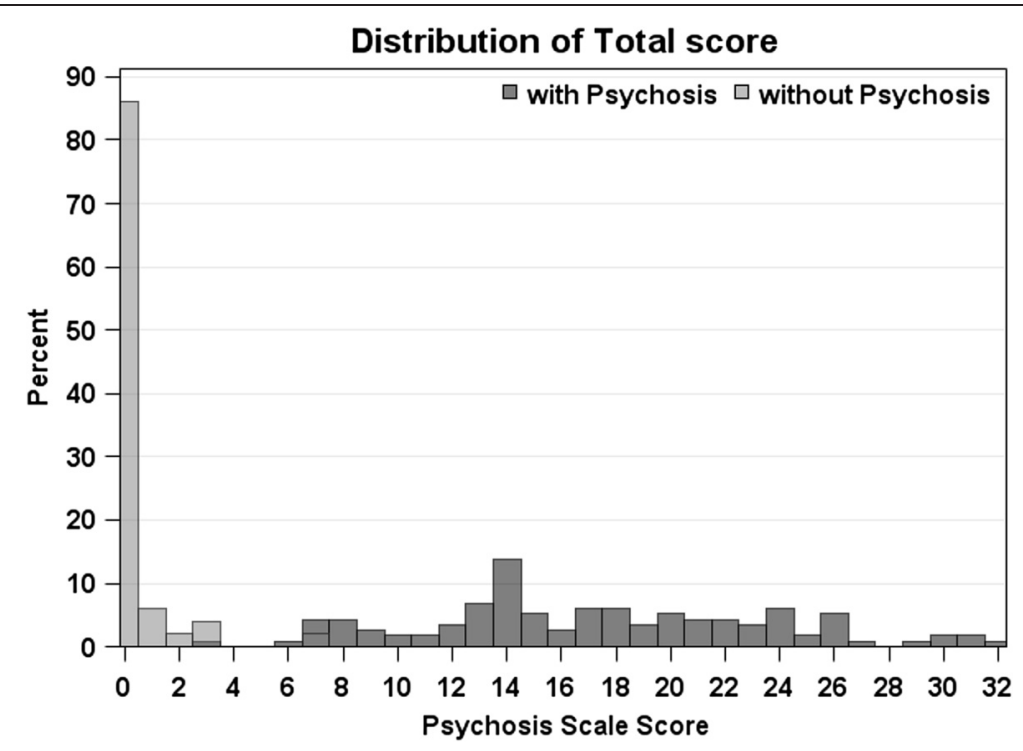

Fig. 2 Histogram of Total Scores $N=100$ scales with psychosis and 50 scales without psychosis

respond similarly to interventions to reduce psychosis (dopamine dose reduction, addition of anti-psychotic medications), suggesting they are intrinsically different and perhaps not best thought of as "hallucinations". It is also our opinion that they do not contribute much to morbidity. "Passage" (very brief) hallucinations/illusions are differentiated from more prolonged visual hallucinations in the duration score. Delusions are usually the most problematic psychosis. We have only a single question on amount of delusions, compared to four regarding different hallucinations, but hypothesized that the final five questions regarding how the psychosis affects the patient would score higher on subjects with delusions, and compensate for the single delusion question. Even if question \#5 (delusions) is excluded from the total score summation, patients who scored $>0$ on $\# 5$ had significantly higher scores $(p<0.001)$. The last question regarding impact on family was included to account for the poor insight many patients possess, the fact that families are often more disturbed by psychosis than the patient, and because some of the major consequences of psychosis, such as nursing home placement, are often

Table 1 Summary of Data

\begin{tabular}{|c|c|c|c|c|c|}
\hline & \multirow{2}{*}{$\begin{array}{l}\text { Mean Score } \\
\text { in Psychosis Subjects, } \\
\text { mean (SD) }\end{array}$} & \multirow[b]{2}{*}{$\begin{array}{l}\text { Number of } \\
\text { questionnaires } \\
\text { in Control } \\
\text { Subjects with } \\
\text { a score of }>0 \\
\text { (out of } 50 \\
\text { assessments) }\end{array}$} & \multicolumn{2}{|c|}{ Inter-Rater Reliability $(95 \% \mathrm{Cl})^{\mathrm{a}}$} & \multirow{2}{*}{$\begin{array}{l}\text { Intra-Rater Reliability }(95 \% \mathrm{Cl})^{\mathrm{b}} \\
\text { Psychosis }(\mathrm{N}=17)\end{array}$} \\
\hline & & & All $(N=71)$ & $\begin{array}{l}\text { Psychosis patients } \\
(N=46)\end{array}$ & \\
\hline 1. Frequency Visual & $3.13(0.98)$ & 1 & $0.85(0.79,0.91)$ & $0.62(0.47,0.77)$ & $0.51(0.23,0.80)$ \\
\hline 2. Frequency Olfactory & $0.29(0.65)$ & 2 & $0.85(0.70,1.00)$ & $0.84(0.66,1.00)$ & $0.66(0.47,0.86)$ \\
\hline 3. Frequency Auditory & $1.16(1.50)$ & 0 & $0.76(0.66,0.85)$ & $0.72(0.60,0.83)$ & $0.68(0.51,0.85)$ \\
\hline 4. Frequency Presence & $2.06(1.41)$ & 4 & $0.79(0.71,0.87)$ & $0.70(0.58,0.82)$ & $0.72(0.54,0.90)$ \\
\hline 5. Delusion Assessment & $0.97(1.41)$ & 2 & $0.64(0.47,0.81)$ & $0.59(0.40,0.45)$ & $0.65(0.33,0.97)$ \\
\hline 6. Duration of Psychosis & $2.66(0.90)$ & 4 & $0.82(0.73,0.90)$ & $0.59(0.42,0.77)$ & $0.46(0.15,0.77)$ \\
\hline 7. Insight & $2.03(0.97)$ & 2 & $0.75(0.65,0.86)$ & $0.58(0.41,0.76)$ & $0.65(0.48,0.82)$ \\
\hline 8. Threatening & $1.34(0.60)$ & 0 & $0.82(0.73,0.91)$ & $0.62(0.43,0.80)$ & $0.77(0.47,1.00)$ \\
\hline 9. Interaction & $1.71(0.82)$ & 0 & $0.81(0.73,0.90)$ & $0.62(0.45,0.80)$ & $0.37(0.07,0.66)$ \\
\hline 10. Family Concern & $1.90(1.42)$ & 0 & $0.86(0.78,0.94)$ & $0.80(0.68,0.92)$ & $0.86(0.73,0.99)$ \\
\hline TOTAL & $17.23(6.30)$ & 7 & $0.92(0.89,0.94)$ & $0.87(0.80,0.92)$ & $0.86(0.80,0.91)$ \\
\hline
\end{tabular}

Weighted kappa statistics for each of 10 specific questions and intra-class correlation coefficients (ICC) for total score were shown as Inter- ${ }^{a}$ and Intra-rater ${ }^{\mathrm{b}}$ reliability 
determined by the caregivers. Since patients themselves have markedly variable insight into their own hallucinations, asking impact of their own psychosis would be difficult to quantify. We also did not exclude patients with varying degrees of cognitive impairment, who may further require family input. We did not formally assess cognition at time of assessment so can't statistically compare "demented" vs "not-demented" subject results.

Our scale has several potential weaknesses. Data on testretest reliability was skewed towards subjects with less severe psychosis, as more severe subjects required immediate interventions, and therefore could not be reassessed for this purpose. We did not compare our results to any validated general psychosis study (content validity) because their content was not designed for the PD psychosis phenotype so any subsequent interpretation of "content" validity would have limited utility. Content validity was excellent based on UPDRS psychosis question (mean 17.23 for score $>0$ vs. 0.36 for 0) We did not formally assess sensitivity to change with intervention, although several subjects started on clozapine showed marked reduction in scores (data not shown).

The final 10 question set was created over a decade and included patient and family input, however they were not formally culled from a larger set and did not undergo cognitive pre-testing. We included subjects with dementia, as this is common in hallucinating patients, so family input was absolutely necessary in this group, as demented subjects could not understand or respond to some of the questions by themselves. No subject had psychosis in the absence of dopaminergic medications, but we did not attempt to exclude the clinical diagnosis of dementia with Lewy bodies, except by onset of motor vs. cognitive symptoms. Importantly, the questionnaire is not meant to be self-administered and some interpretation of response is needed by the interviewer (discussed more fully in the Additional file 1: Instructions), nor is it meant to be a screening tool to diagnose psychosis. Future research could formally assess sensitivity to treatment response, correlations with other scales assessing quality of life, other scales for psychosis, formal comparison of demented vs. non-demented patients, comparison of patient vs family scores, and neurophysiology correlates.

\section{Conclusion}

We feel this scale offeres very good content valisity, inter- and intra-rater reliability and ease of use.

\section{Additional file}

Additional file 1: Instructions for completing the psychosis scale.

(DOCX $17 \mathrm{~kb})$

\section{Competing interest}

There was no specific funding for this project. There are no conflicts of interest related to this article.

William Ondo: speaker- Lundbeck, TEVA, Merz, UCBPharma, Avanir, IMPAX advisory board - Lundbeck, Auspex, UCBPharma, Abbvie, ACADIA

Sana Sarfaraz, BA none

MinJae Lee, PhD none

\section{Authors' contribution}

WO, All aspects. SS, data acquisition and critical review. ML statistical analysis and critical review. All authors read and approved the final manuscript.

\section{Author details}

${ }^{1}$ Department of Neurology, Methodist Neurological Institute, 6560 Fannin, Ste 802, Houston, TX 77030, USA. ${ }^{2}$ Biostatistics/Epidemiology/Research Design (BERD) Core, Center for Clinical and Translational Sciences, The University of Texas Health Science Center at Houston and Division of Clinical and Translational Sciences, Houston, TX 77030, USA. ${ }^{3}$ Department of Internal Medicine, The University of Texas Medical School at Houston Center for Clinical and Translational Sciences, University of Texas Health Science Center at Houston, Houston, TX 77030, USA.

Received: 19 June 2015 Accepted: 17 August 2015

Published online: 01 December 2015

\section{References}

1. Friedman JH. Parkinson's disease psychosis 2010: a review article. Parkinsonism Relat Disord. 2010;16(9):553-60. doi:10.1016/ j.parkreldis.2010.05.004

2. Goetz CG. Scales to evaluate psychosis in Parkinson's disease. Parkinsonism Relat Disord. 2009;15 Suppl 3:S38-41. doi:10.1016/S1353-8020(09)70777-1.

3. Friedberg G, Zoldan J, Weizman A, Melamed E. Parkinson Psychosis Rating Scale: a practical instrument for grading psychosis in Parkinson's disease. Clin Neuropharmacol. 1998;21(5):280-4.

4. Brandstaedter D, Spieker S, Ulm G, Siebert U, Eichhorn TE, Krieg JC, et al. Development and evaluation of the Parkinson Psychosis Questionnaire A screening-instrument for the early diagnosis of drug-induced psychosis in Parkinson's disease. J Neurol. 2005;252(9):1060-6. doi:10.1007/s00415-0050816-x.

5. Cummings $J$ L, Mega M, Gray K, Rosenberg-Thompson S, Carusi DA, Gornbein J. The Neuropsychiatric Inventory: comprehensive assessment of psychopathology in dementia. Neurology. 1994;44(12):2308-14.

6. Kay SR, Opler LA, Lindenmayer JP. The Positive and Negative Syndrome Scale (PANSS): rationale and standardisation. Br J Psychiatry Suppl. 1989;7:59-67.

7. Overall JE, Gorham DR. The brief psychiatric rating scale. Psychol Rep. 1962:10:799-812

8. Anonymous. Low-dose clozapine for the treatment of drug-induced psychosis in Parkinson's disease. The Parkinson Study Group. Eng J Med. 1999;340(10):757-63.

9. Cummings J, Isaacson S, Mills R, Williams H, Chi-Burris K, Corbett A, et al. Pimavanserin for patients with Parkinson's disease psychosis: a randomised, placebo-controlled phase 3 trial. Lancet. 2014;383(9916):533-40. doi:10.1016/ S0140-6736(13)62106-6.

10. Ondo WG, Levy JK, Vuong KD, Hunter C, Jankovic J. Olanzapine treatment for dopaminergic-induced hallucinations. Mov Disord. 2002;17(5):1031-5. doi:10.1002/mds.10217.

11. Ondo WG, Tintner R, Voung KD, Lai D, Ringholz G. Double-blind, placebo-controlled, unforced titration parallel trial of quetiapine for dopaminergic-induced hallucinations in Parkinson's disease. Mov Disord. 2005;20(8):958-63.

12. McKeith I, Mintzer J, Aarsland D, Burn D, Chiu H, Cohen-Mansfield J, et al. Dementia with Lewy bodies. Lancet Neurol. 2004;3(1):19-28.

13. Chou KL, Messing S, Oakes D, Feldman PD, Breier A, Friedman JH. Drug-induced psychosis in Parkinson disease: phenomenology and correlations among psychosis rating instruments. Clin Neuropharmacol. 2005;28(5):215-9.

14. Gallagher DA, Parkkinen L, O'Sullivan SS, Spratt A, Shah A, Davey CC, et al. Testing an aetiological model of visual hallucinations in Parkinson's disease. Brain. 2011;134(Pt 11):3299-309. doi:10.1093/brain/awr225. 